\title{
Las normativas de construcción con tierra en el mundo
}

\author{
The earth building normative documents in the world
}

L. Cid, F. R. Mazarrón, I. Cañas ${ }^{(*)}$

\section{RESUMEN}

La tierra ha sido utilizada como material de construcción desde hace siglos. No obstante, la normativa al respecto está muy dispersa, y en la mayoría de países desarrollados surgen numerosos problemas técnicos y legales para llevar a cabo una construcción con este material. Este artículo estudia el panorama normativo para las construcciones con tierra cruda a nivel internacional, analizando cincuenta y cinco normas y reglamentos de países repartidos por los cinco continentes, que representan el estado del arte de la normalización de la tierra cruda como material de construcción. Es un estudio referenciado sobre las normas y reglamentos vigentes desarrollados por los organismos nacionales de normalización o autoridades correspondientes. Se presentan las normativas y los organismos que las emiten, analizando la estructura y contenido de cada una. Se estudian y analizan los aspectos más relevantes, como la estabilización, selección de los suelos, requisitos de los productos y ensayos existentes, comparando las diferentes normativas. Este trabajo puede ser de gran utilidad para el desarrollo de futuras normas $y$ como referencia para arquitectos e ingenieros que trabajen con tierra.

$113-110$

Palabras clave: normas, edificación con tierra, bloques de tierra comprimida, adobe, tapial.

\section{SUMMARY}

For centuries, earth has been used as a construction material. Nevertheless, the normative in this matter is very scattered, and in the most developed countries, carrying out a construction with this material implies a variety of technical and legal problems. This article analyzes, in an international level, the normative panorama about constructions with earth, analyzing fifty five standards and regulations of countries all around the five continents; these represent the state of art that normalizes the earth as a construction material. It is a study indexed on the actual procedures and regulations developed by the national organisms of normalization or correspondent authorities. The standards and the organisms that produce them appear, analyzing the structure and the content of each one. We have studied and analyzed the most relevant aspects, such as stabilization, soil selections, the requisites of the products and the existent test, comparing the diverse normative. The knowledge from this study could be very useful for the development of future standards and as a reference for architects and engineers that work with earth.

Keywords: standards, earth building, compressed earth blocks, adobe, rammed earth.

\footnotetext{
(*) Universidad Politécnica de Madrid (España) 


\section{INTRODUCCIÓN}

La tierra es cada vez más valorada como material de construcción. Dado el creciente interés por este antiguo material, y ante la falta de un marco legal muchos países intentan normalizar su uso para resolver los problemas actuales derivados de la ausencia de una normativa que permita el uso de las técnicas de construcción con tierra cruda.

Son numerosos los trabajos que se centran en el estudio de normas y reglamentos en el ámbito de la construcción como por ejemplo los estudios realizados por Soronis (1), Cañas (2), Hooton (3) o Mahlia (4). En el campo de aplicación de la edificación con tierra son muchos los países que en los últimos años trabajan en la normalización destacan Colombia (2005) y España (2008), con la publicación de nuevas normas; Chile, Ecuador, México y Nicaragua, desarrollando futuras normas; o Perú mejorando documentos ya existentes (5).

Esta creciente actividad hace conveniente analizar el estado del arte de la normalización para la construcción con tierra cruda. Para ello hemos llevado a cabo una búsqueda en diversidad de fuentes, organismos internacionales de normalización, bases de datos, organizaciones y redes de construcción con tierra, además de basarnos en listas de normativas ya publicadas (2).

Siguiendo la clasificación realizada por Cañas 2007 (2), el estudio se ha centrado en las normas emitidas por los organismos nacionales de normalización y los reglamentos emitidos por las autoridades, según la Norma Europea EN 45020: 2007 que a su vez adopta la Guía ISO/IEC 2:2004 (6). No obstante aunque no sea objeto de estudio en este trabajo, es importante señalar la existencia de documentos que sin tener el carácter de normas o reglamentos, tienen importancia en ciertos países como es el caso de los documentos normativos de Alemania o Australia.

\section{NORMAS Y REGLAMENTOS}

En el proceso de búsqueda y estudio de normas y reglamentos internacionales del marco de la construcción con tierra cruda se han localizado 55 documentos. En la tabla 1 se detallan las normas o reglamentos encontrados, donde se indica: País, la referencia de la Norma o del Reglamento; el organismo que la emite (ORG); la referencia bibliografía, que queda reflejada por el número que aparece en el epígrafe de bibliografía del presente artículo (REF); si el documento sólo trata la tierra estabilizada (EST), y si contempla alguna de las tres principales técnicas en las que se centra nuestro estudio: adobe, tapial o bloque comprimido (Técnica). En la tabla 2 se encuentran ordenados por fecha de publicación de la normativa vigente de cada país.

El conjunto de documentos encontrados es muy variado. Para su mejor manejo y comprensión, se han agrupado algunas normas de un mismo país. Son los casos de las normas brasileñas para BTC (7-19), las neozelandesas (32-34), las peruanas NTP de adobe estabilizado (36-38), la colección del organismo regional de normalización africano ARS 670-683 (3952) y las normas de Túnez $(56,57)$.

De todos ellos se han seleccionado un conjunto de normas y reglamentos que son representativos de la normativa de edificación con tierra cruda a nivel internacional y válidos para detallar el estado actual (ver tabla 3). En esta selección se han descartado la norma nigeriana NIS 369 (31) por la dificultad para acceder y conseguir el documento; las normas turcas TS 537, TS 2514 y TS 2515 (58-60) por no estar redactadas en español, inglés o francés; y las leyes italianas $(28,29)$ atendiendo a su escasa aplicabilidad con carácter técnico-constructivo.

Esta selección se estructura en quince grupos de normas y reglamentos, (contabilizando en un mismo grupo todas las normas o reglamentos de un país excepto en el caso de Perú y EEUU por haberse desarrollado por entidades diferentes). Todas han sido emitidas por organismos nacionales, excepto una norma estatal de Nuevo México, el grupo de normas internacionales de un organismo regional africano (ARSO), que comprende varios países de África y la norma publicada por el Organismo ASTM Internacional. En la figura 1 se muestra por orden cronológico las normas y reglamentos por año de aprobación.

\section{Grupo 1: Brasil}

Brasil ha emitido trece normas (7-19), desarrolladas por la Asociación Brasileña de Normas Técnicas (ABNT) desde 1986 hasta 1996, sobre el suelo cemento y sus aplicaciones constructivas en forma de bloque suelo cemento y pared monolítica.

\section{Grupo 2: Colombia}

En 2005 se emite la norma colombiana NTC 5324 (20), editada por ICONTEC, siendo una traducción de la norma experimental francesa XP P13-901,2001(24) de AFNOR sobre BTC.

Grupo 3-4: EEUU

El reglamento de Nuevo México (21) lo emite el CID (Construction Industries Division) en 2004, basado en dos códigos actualmente derogados 
Recientemente, la organización internacional "American Society for Testing and Materials" ha desarrollo la norma técnica ASTM E2392 M-10 (22) aprobada en enero del 2010 y publicada en marzo de ese año.

\section{Grupo 5: España}

A finales de 2008 se desarrolla la primera norma española (23) de construcción en tierra, y primera norma europea actual no experimental para bloques de tierra comprimida, emitida por el subcomité AEN/CTN 41 SC 10 "Edificación con tierra cruda" de AENOR.

\section{Grupo 6: Francia}

La norma experimental XP P13-901:2001 (24) es un documento provisional y accesible al público, desarrollado por el organismo nacional AFNOR con el objeto de poder obtener la necesaria experiencia en su aplicación, sobre la cual basar una futura norma

Grupo 7: India

El organismo regulador de la India desde 1987 es el BIS (Bureau of Indian Standards) cuyo antecesor fue el ISI (Indian Standards Institution) que publicó las normas IS 2110 (25) revisada en mayo del 2007, la norma IS 1725 (26) y la norma IS 13827 (27).

Grupo 8: Kenya

La KS 02-1070 (30) emitida por el KBS (Kenya Bureau of Standards) es una revisión de la norma de 1993.

Grupo 9: Nueva Zelanda

En 1996 se publicaron las tres normas emitidas por el organismo nacional SNZ (New Zealand Standards) (32-34)

\section{Grupo 10-11: Perú}

La norma peruana NTE E 080 (35) del 2000 procede de una versión anterior de 1977 del ININVI (Instituto Nacional de Investigación y Normalización de la Vivienda) que fue ab- sorbido por el organismo público SENCICO (Servicio Nacional de Normalización, Capacitación e Investigación para la Industria de la Construcción). Actualmente la norma peruana se encuentra en una segunda revisión y ampliación. Las normas NTP (36-38), emitidas por el sistema Peruano de Normalización INDECOPI.

\section{Grupo 12: Regional África}

En 1998 se emiten 14 normas sobre BTC (3952) de la organización regional de normalización africana, (ARSO) publicadas en una serie tecnológica del CDI/CRATerre (60).

Grupo 13: Sri Lanka

El instituto de normalización de Sri Lanka (SLSI) desarrolla a finales del 2009 tres normas sobre los bloques de tierra comprimida estabilizados (53-55).

\section{Grupo 14: Túnez}

Dos normas NT $(56,57)$ publicadas en 1996 por el organismo normalizador de Túnez, INNORPI.

\section{Grupo 15: Zimbabue}

La norma de Zimbabwe (61) fue emitida en 2001 por el organismo nacional de Normalización (SAZ) y se basa en una publicación de Keable 1996 (62).

\section{ANÁLISIS DE LA ESTRUCTURA Y CONTENIDO DE LA NORMATIVA INTERNACIONAL}

Una gran parte de los documentos analizados $(79 \%)$ centran su contenido en el estudio de una única técnica constructiva, ya sea adobe, BTC o tapial. Los restantes documentos examinan varias técnicas. De esta forma las normas SAZS 724 (61) e IS 2110 (25) son exclusivas para tapial. Conteniendo disposiciones únicamente para adobe se encuentra la peruana NTE E 080 (35) y, para adobe es-

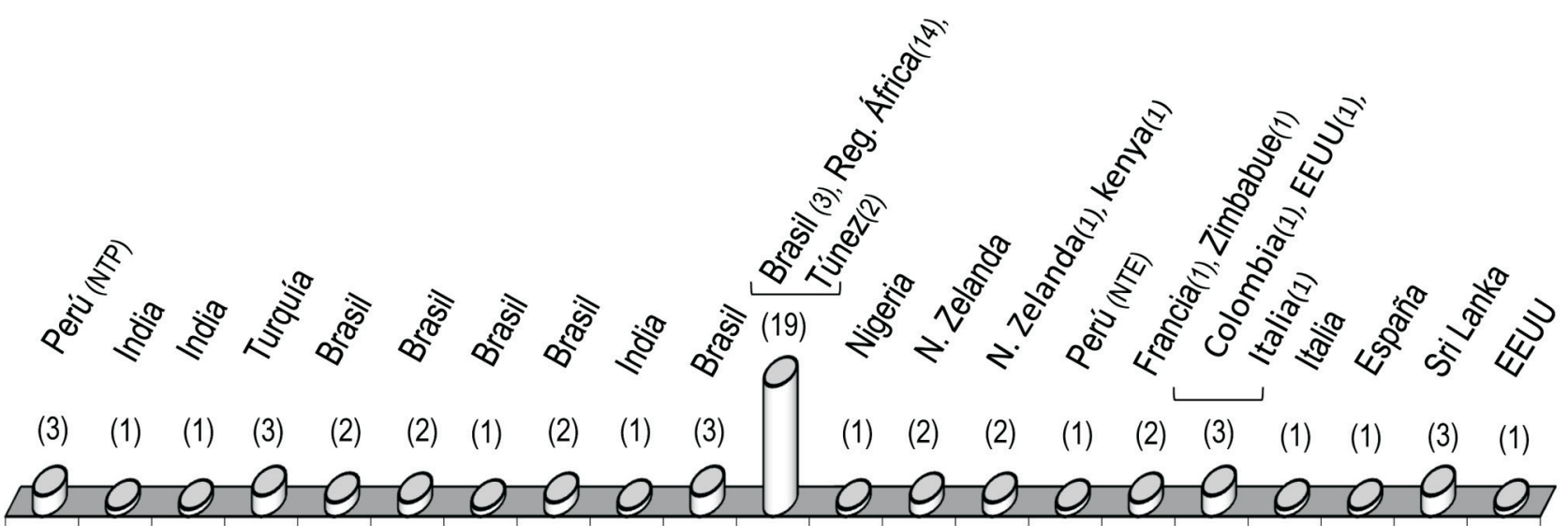

197919801982198519861989199019921993199419961997199819992000200120042006200820092010

$\left(\mathrm{N}^{\circ}\right)$ : número de normas vigentes entre paréntesis. 
Tabla 1

Listado de normas y reglamentos encontrados

\begin{tabular}{|c|c|c|c|c|c|c|c|c|}
\hline País & Norma/Reglamento & ORG & REF & EST & $\frac{8}{8}$ & U. & \begin{tabular}{|l|}
$\frac{\pi}{2}$ \\
$\frac{\pi}{\circ}$ \\
\end{tabular} & Notas \\
\hline \multirow{13}{*}{ Brasil } & NBR 8491, 1986. & \multirow{13}{*}{ ABNT } & 7 & \multirow{13}{*}{$\mathrm{x}$} & & \multirow{7}{*}{$x$} & \multirow{2}{*}{\multicolumn{2}{|c|}{$\begin{array}{l}\text { BTC estabilizado con cemento especifica, } \\
\text { métodos de ensayo. }\end{array}$}} \\
\hline & NBR 8492, 1986. & & 8 & & & & & \\
\hline & NBR 10832, 1989 & & 9 & & & & & \multirow{2}{*}{$\begin{array}{l}\text { Procedimiento fabricación BTC con } \\
\text { prensa manual/hidráulica }\end{array}$} \\
\hline & NBR 10833, 1989 & & 10 & & & & & \\
\hline & NBR 10834, 1994. & & 11 & & & & & \multirow{3}{*}{$\begin{array}{l}\text { Especificaciones y métodos de ensayo de } \\
\text { bloques de suelo- cemento }\end{array}$} \\
\hline & NBR 10835, 1994 & & 12 & & & & & \\
\hline & NBR 10836, 1994 & & 13 & & & & & \\
\hline & NBR 12023, 1992 & & 14 & & & & & \multirow{5}{*}{ Métodos de ensayo para suelo-cemento } \\
\hline & NBR 12024, 1992 & & 15 & & & & & \\
\hline & NBR 12025, 1990 & & 16 & & & & & \\
\hline & NBR 13554, 1996 & & 17 & & & & & \\
\hline & NBR 13555,1996 & & 18 & & & & & \\
\hline & NBR 13553, 1996 & & 19 & & & & $\mathrm{x}$ & Tapia con cemento \\
\hline Colombia & NTC 5324,2004 & ICONTEC & 20 & $x$ & & $\mathrm{x}$ & & Estabilizado con cemento \\
\hline \multirow{2}{*}{ EEUU } & NMAC, 14.7.4, 2004 & CID & 21 & & $\mathrm{x}$ & $\mathrm{x}$ & $\mathrm{x}$ & Reglam. Estatal de Nuevo México. \\
\hline & ASTM E2392 M-10 & ASTM & 22 & & $x$ & & $\mathrm{x}$ & \\
\hline España & UNE 41410:2008 & AENOR & 23 & & & $x$ & & Primera norma Europea \\
\hline Francia & XP P13-901,2001 & AFNOR & 24 & & & $\mathrm{x}$ & & Norma experimental \\
\hline \multirow{3}{*}{ India } & IS $2110: 1980$ & BIS & 25 & $\mathrm{x}$ & & 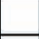 & $\mathrm{x}$ & Paredes de suelo-cemento \\
\hline & IS $1725: 1982$. & BIS & 26 & $x$ & & $x$ & & \\
\hline & IS $13827: 1993$ & BIS & 27 & & $\mathrm{x}$ & & $\mathrm{x}$ & Directrices resistencia a terremotos \\
\hline \multirow{2}{*}{ Italia } & Ley no 378,2004 & & 28 & & \multirow{2}{*}{$\mathrm{x}$} & \multirow{2}{*}{$x$} & \multirow{2}{*}{$\mathrm{X}$} & \multirow{2}{*}{$\begin{array}{l}\text { Leyes para la conservación del } \\
\text { patrimonio de tierra }\end{array}$} \\
\hline & L.R. 2/062 2006 & & 29 & & & & & \\
\hline Kenya & KS 02-1070: 1999. & KEBS & 30 & $\mathrm{x}$ & & $x$ & & \\
\hline Nigeria & NIS 369:1997. & SON & 31 & $\mathrm{x}$ & & $x$ & & \\
\hline & NZS 4297, 1998. & & 32 & & & & & \\
\hline $\begin{array}{l}\text { Nueva } \\
\text { Zelanda }\end{array}$ & NZS 4298, 1998. & SNZ & 33 & & $x$ & $x$ & $x$ & \\
\hline & NZS 4299, 1999. & & 34 & & & & & \\
\hline & NTE E 0.80, 2000 & SENCICO & 35 & & $\mathrm{x}$ & & & \\
\hline Perú & NTP $331.201,1979$ & & 36 & & & & & \\
\hline retu & NTP 331.202, 1979. & $\begin{array}{c}1 \\
\text { INDECOP }\end{array}$ & 37 & $x$ & $x$ & & & \\
\hline & NTP $331.203,1979$. & & 38 & & & & & \\
\hline & ARS 670, 1996 & & 39 & & & & & \\
\hline & ARS 671,1996 & & 40 & & & & & \\
\hline & ARS 672,1996 & & 41 & & & & & \\
\hline & ARS 673, 1996 & & 42 & & & & & \\
\hline & ARS 674, 1996 & & 43 & & & & & \\
\hline & ARS 675, 1996 & & 44 & & & & & \\
\hline Regional & ARS 676, 1996 & ARSO & 45 & & & $x$ & & \\
\hline África & ARS 677,1996 & & 46 & & & 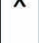 & & \\
\hline & ARS 678, 1996 & & 47 & & & & & \\
\hline & ARS 679, 1996 & & 48 & & & & & \\
\hline & ARS 680, 1996 & & 49 & & & & & \\
\hline & ARS 681, 1996 & & 50 & & & & & \\
\hline & ARS 682, 1996 & & 51 & & & & & \\
\hline & ARS 683, 1996 & & 52 & & & & & \\
\hline Sri Lanka & SLS 1382-1:2009 & SLSI & 53 & $\mathrm{x}$ & & $x$ & & Bloques de suelo comprimido \\
\hline & SLS 1382-2:2009 & & 54 & & & & & estabilizados \\
\hline & SLS 1382-3:2009 & & 55 & & & & & \\
\hline Túnez & NT 21.33:1996 & INNORPI & 56 & & & $y$ & & En francés \\
\hline 7umez & NT 21.35:1996 & I IVIVUnt & 57 & & & x & & En francés \\
\hline & TS 537, 1985. & & 58 & $x$ & & & & \\
\hline Turquía & TS 2514, 1985. & TSE & 59 & & $x$ & & & En turco \\
\hline & TS 2515, 1985. & & 60 & & & & & \\
\hline Zimbabue & SAZS 724, 2001. & SAZ & 61 & & & & $\mathrm{x}$ & \\
\hline
\end{tabular}

Abreviaturas: ORG (Organismo); REF (Referencia, ver en bibliografía el número); EST (Estabilización, si solo contempla el uso de tierra estabilizada) 
Tabla 2

Cuadro de normativa vigente

\begin{tabular}{|c|c|c|c|}
\hline Año & País & Norma & REF \\
\hline 1979 & Perú & $\begin{array}{l}\text { NTP } \\
331.201,331.202,331.203\end{array}$ & $36-38$ \\
\hline 1980 & India & IS 2110 & 25 \\
\hline 1982 & India & IS 1725 & 26 \\
\hline 1985 & Turquía & TS 537, TS 2514, TS 2515 & $58-60$ \\
\hline 1986 & Brasil & NBR 8491,8492 & $7-8$ \\
\hline 1989 & Brasil & NBR 10832,10833 & 9-10 \\
\hline 1990 & Brasil & NBR 12025 & 16 \\
\hline 1992 & Brasil & NBR 12023,12024 & $14-15$ \\
\hline 1993 & India & IS $13827: 1993$ & 27 \\
\hline 1994 & Brasil & NBR $10834,10835,10836$ & $11-13$ \\
\hline \multirow{3}{*}{1996} & Brasil & NBR $13554,13555,13553$ & 17-19 \\
\hline & $\begin{array}{l}\text { Regional } \\
\text { África }\end{array}$ & ARS 670-683 & $39-52$ \\
\hline & Túnez & NT $21.33,21.35$ & $56-57$ \\
\hline 1997 & Nigeria & NIS 369 & 31 \\
\hline 1998 & $\begin{array}{l}\text { Nueva } \\
\text { Zelanda }\end{array}$ & NZS 4297, 4298 & $32-33$ \\
\hline \multirow[t]{2}{*}{1999} & $\begin{array}{l}\text { Nueva } \\
\text { Zelanda }\end{array}$ & NZS 4299 & 34 \\
\hline & Kenya & KS $02-1070$ & 30 \\
\hline 2000 & Perú & NTE E 0.80 & 35 \\
\hline \multirow{2}{*}{2001} & Francia & XP P13-901 & 24 \\
\hline & Zimbabue & SAZS 724 & 61 \\
\hline \multirow{3}{*}{2004} & Colombia & NTC 5324 & 20 \\
\hline & EEUU & NMAC, 14.7.4 & 21 \\
\hline & Italia & Ley $n=378,2004$ & 28 \\
\hline 2006 & Italia & L.R. $2 / 06$ & 29 \\
\hline 2008 & España & UNE 41410 & 23 \\
\hline 2009 & Sri Lanka & SLS 1382-1, 1382-2, 1382-3, & $53-55$ \\
\hline 2010 & EEUU & ASTM E2392 M-10 & 61 \\
\hline
\end{tabular}

tabilizado con asfalto, la NTP 331.201, NTP 331.202 y NTP 331.203 (36-38). Regulando sólo el bloque de tierra comprimido se haIlan la francesa XP P13-901 (24), la colombiana NTC 5324 (20), la keniata KS 02-1070 (30), la colección de la regional africana ARS 670 a ARS 683 (39-52), las NT 21.33 y NT $21.35(56,57)$ y la norma española UNE 41410 (23).

La brasileña es exclusivamente de bloque de tierra comprimido, excepto la norma NBR 13553 (19) que comprende pared monolítica. Las normas indias IS 2110 e IS $1725(25,26)$ regulan el tapial (paredes de suelo-cemento) y el BTC respectivamente. La NMAC 14.7.4 (21) trata fundamentalmente las tres técnicas principales, aunque también incluye quemados y terrones. La más completa de las trece es la neozelandesa, colección formada por las NZS 4297, NZS 4298, NZS 4299 (32-
34), que comprende adobe, bloque de tierra comprimido y tapial, así como tierra vertida. Sólo de forma informativa ofrece también recomendaciones para cob y adobe in-situ. Como se observa, sólo dos tratan a la vez las tres técnicas principales, NMAC 14.7.4 (21) y las NZS (32-34). Es de resaltar que de los quince grupo de normas, once dedican parte o la totalidad de su norma al bloque de tierra comprimido.

En cuanto a contenido, podemos decir que el documento más amplio y completo es el trío neozelandés (32-34), pues comprende desde requisitos de materiales y construcción a cuestiones de diseño estructural y de durabilidad de los edificaciones de tierra, y tanto para adobe como para bloque de tierra comprimido o tapial. El contenido de este grupo de normas es algo más reducido en cuanto a fabricación de las unidades, tema 
Tabla 3

Normas y reglamentos seleccionados y contenido

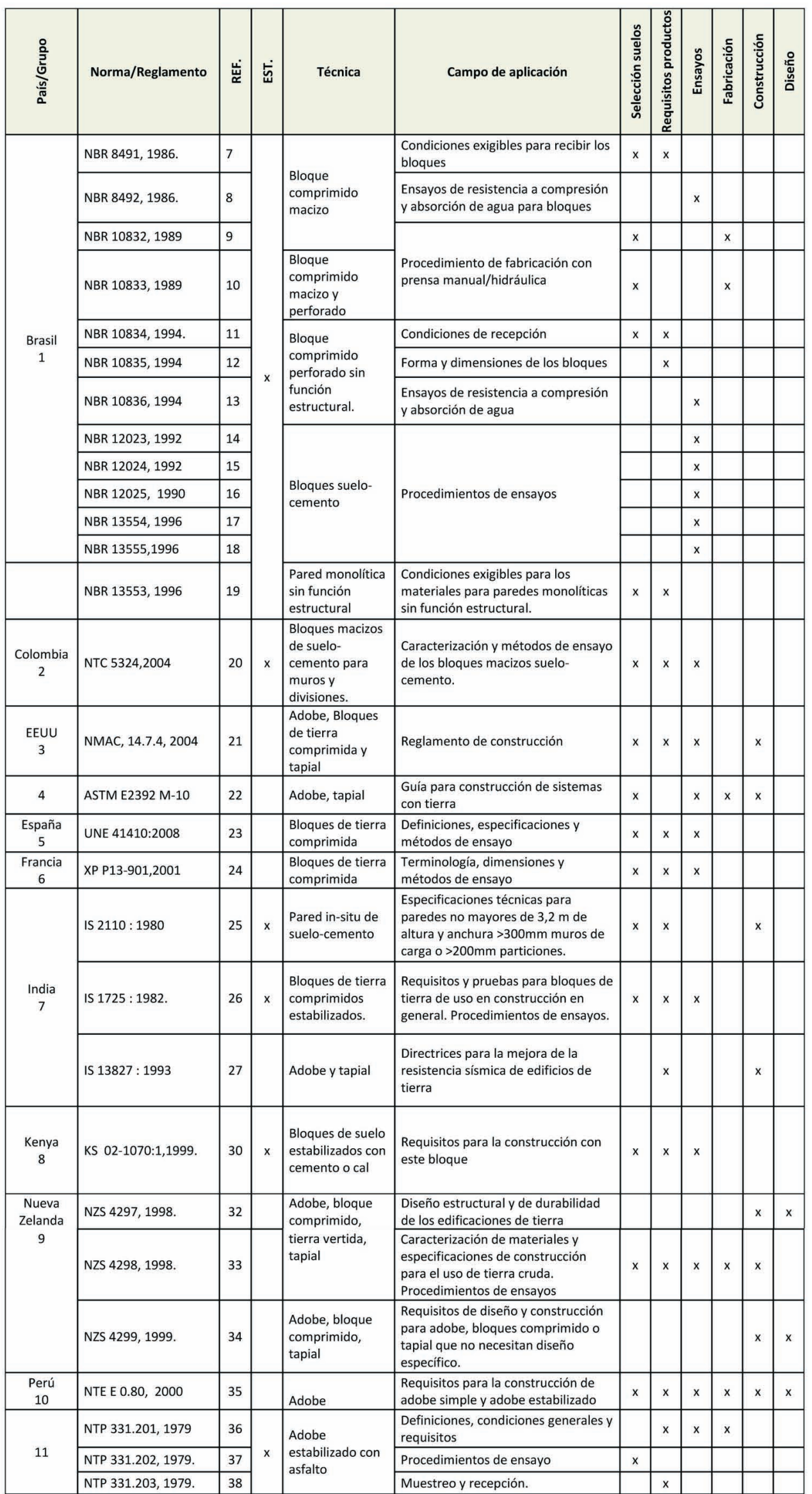


Tabla 3

Normas y reglamentos seleccionados y contenido

\begin{tabular}{|c|c|c|c|c|c|c|c|c|c|c|c|}
\hline 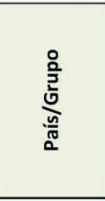 & Norma/Reglamento & 岀 & 点 & Técnica & Campo de aplicación & 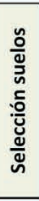 & 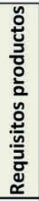 & 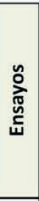 & 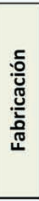 & 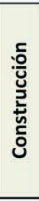 & 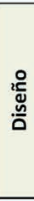 \\
\hline \multirow{14}{*}{$\begin{array}{c}\text { Regional } \\
\text { África } \\
12\end{array}$} & ARS 670, 1996 & 39 & & \multirow{14}{*}{$\begin{array}{l}\text { Bloques de tierra } \\
\text { comprimida }\end{array}$} & Terminología BTC. & $\mathrm{x}$ & & & & & \\
\hline & ARS 671, 1996 & 40 & & & Clasificar los BTC & & & & & & \\
\hline & ARS 672, 1996 & 41 & & & Clasificación de morteros de tierra. & & & & & & \\
\hline & ARS 673, 1996 & 42 & & & Definir formas de albañilería & & & & & & \\
\hline & ARS 674, 1996 & 43 & & & Requisitos de BTC ordinarios & $\mathrm{x}$ & $\mathrm{x}$ & & & & \\
\hline & ARS 675, 1996 & 44 & & & Requisitos aplicables a BTC vistos & $\mathrm{x}$ & $x$ & & & & \\
\hline & ARS 676, 1996 & 45 & & & Requisitos de morteros ordinarios & & $\mathrm{x}$ & & & & \\
\hline & ARS 677, 1996 & 46 & & & Requisitos de morteros vistos & & $\mathrm{x}$ & $\mathrm{x}$ & & & \\
\hline & ARS 678, 1996 & 47 & & & Requisitos para albañilería revestida & & & & & $\mathrm{x}$ & $\mathrm{x}$ \\
\hline & ARS 679, 1996 & 48 & & & Requisitos para albañilería vista & & & & & $\mathrm{x}$ & $\mathrm{x}$ \\
\hline & ARS 680, 1996 & 49 & & & Estado del arte fabricación BTC & $\mathrm{x}$ & & & $\mathrm{x}$ & & \\
\hline & ARS 681, 1996 & 50 & & & Estado del arte para morteros tierra. & $\mathrm{x}$ & & & $\mathrm{x}$ & & \\
\hline & ARS 682, 1996 & 51 & & & Estado del arte para construcción & & & & & $\mathrm{x}$ & \\
\hline & ARS 683, 1996 & 52 & & & Pruebas requeridas & $\mathrm{x}$ & $x$ & & & & \\
\hline \multirow{3}{*}{$\begin{array}{l}\text { Sri Lanka } \\
13\end{array}$} & SLS 1382-1:2009 & 53 & \multirow{3}{*}{$\mathrm{x}$} & $\begin{array}{l}\text { Bloques de tierra } \\
\text { comprimida }\end{array}$ & Requerimientos & $\mathrm{x}$ & $\mathrm{x}$ & & & & \\
\hline & SLS 1382-2:2009 & 54 & & $\begin{array}{l}\text { Bloques de tierra } \\
\text { comprimida }\end{array}$ & Métodos de ensayo & & & $\mathrm{x}$ & & & \\
\hline & SLS 1382-3:2009 & 55 & & $\begin{array}{l}\text { Bloques de tierra } \\
\text { comprimida }\end{array}$ & $\begin{array}{l}\text { Guía sobre producción, diseño y } \\
\text { construcción }\end{array}$ & & & & $\mathrm{x}$ & $\mathrm{x}$ & $\mathrm{x}$ \\
\hline \multirow[t]{2}{*}{$\begin{array}{c}\text { Túnez } \\
14\end{array}$} & |NT 21.33:1996 & 56 & & \multirow[t]{2}{*}{$\begin{array}{l}\text { Bloques de tierra } \\
\text { comprimida }\end{array}$} & $\begin{array}{l}\text { Especificaciones para BTC ordinarios, } \\
\text { características geométricas, fisico- } \\
\text { quimicas... } \\
\end{array}$ & & $x$ & $x$ & & & \\
\hline & NT 21.35:1996 & 57 & & & Definición y clasificación de BTC & & $x$ & & & & \\
\hline $\begin{array}{c}\text { Zimbabue } \\
15\end{array}$ & SAZS 724, 2001. & 61 & & Tapial & $\begin{array}{l}\text { Guías para el diseño, construcción y } \\
\text { ensayos para estructuras de tapial. }\end{array}$ & $\mathrm{x}$ & $\mathrm{x}$ & $\mathrm{x}$ & & $x$ & $\mathrm{x}$ \\
\hline
\end{tabular}

que se halla en las brasileñas (9-10) y la ARS 680 (49) para el bloque de tierra comprimido. Disposiciones sobre construcción y ejecución aparecen con detalle únicamente en la NMAC 14.7.4 (21) de Nuevo México, la SAZS 724 (61) de Zimbabue, la NTE E 0.80 (35) de Perú, la NZS 4298 (33) de Nueva Zelanda y la africana ARS 682 (51).

Las que más contenido dedican al diseño estructural son de nuevo las neozelandesas $(32,33)$, usándose en su formulación principios de diseño de estados limite. Limitaciones de esbeltez de muros e indicaciones sobre sus arriostramientos se indican en el reglamento de Nuevo MéxiCo, NMAC 14.7.4 (21); en la de Zimbabue, SAZS 724 (61) y en la NTE E 0.80 (35) de Perú. Lo que en todas se encuentra son requisitos que deben exigirse a los productos, por medio de resultados mínimos que deben obtenerse de una serie de ensayos. Esta serie de ensayos en todos los casos aparecen explicados en las propias normas o referenciados a otros documentos que contienen sus procedimientos. Sólo en el caso de NMAC 14.7.4 (21) no se halla referencia alguna.

En todas las normas se contempla fundamentalmente el empleo de los muros de tierra como muros resistentes, que además podrían utilizarse como no resistentes. So- lamente las normas brasileñas $(11-13,19)$ ponen limitaciones al uso de bloques huecos y pared monolítica sin función estructural.

Respecto al campo de aplicación de estas técnicas de tierra, algunas normas limitan la altura para muros de carga a dos plantas como máximo (21) o no debiendo exceder los 6,5 m de altura desde la parte superior de la cimentación hasta la parte alta del muro de tierra (30). En algunas ocasiones se establecen categorías de edificios como en las normas NZS (32-34), categoría normal una o dos alturas cuyo requisito son unas propiedades mínimas de uso, superficie, altura de muros, espesores, carga, etc y una. Y una categoría especial que exceden todas las características de la categoría normal

En lo referente a zonas susceptibles a la sismicidad, la norma peruana NTE E 0.80 (35) limita el número de alturas, encontrando también algunas indicaciones para diseño sismoresistente en la neozelandesas (32-34).

\section{ASPECTOS RELEVANTES DE LA NORMATIVA}

\subsection{Estabilización}

En el conjunto de normas seleccionadas se recomienda agregar algún agente estabilizador (cemento, cal...) para mejorar las carac- 
terísticas mecánicas, de durabilidad y de estabilidad de la tierra aunque no es siempre imprescindible, ya que muchos suelos pueden alcanzar un comportamiento satisfactorio sin necesidad de ningún estabilizante. Los estabilizantes más comunes son el cemento $(21,35,39,61)$, cal no hidráulica e hidratada $(33,61)$, o las emulsiones asfálticas para el adobe (35).

Algunas de las normas tienen como objeto de normalización exclusivamente la tierra estabilizada caso de las normas brasileñas (7-19), norma colombiana (20), India (26), Kenya (30) o de las tres normas de Sri Lanka para los bloques de tierra comprimida; para adobe las normas NTP de Perú (36-38) y para tapial la norma brasileña (19) junto con la norma IS 2110 (25).

Otro de los enfoques respecto al uso de estabilizantes es fijar un límite de contenido de estabilizantes, como es el caso de las normas neozelandesas (32-34) y la norma española (23). En el caso de la norma española el contenido total de estabilizantes (cemento, cal, yeso y otros) debe ser menor o igual al $15 \%$ de la masa en seco del bloque. Salvo la norma estadounidense (21) que establece superar un valor de absorción de agua para el caso del adobe, y para tapial contener un mínimo de $6 \%$ en peso de cemento Portland superando las pruebas de resistencia a compresión en húmedo, ningún otro documento define la tierra estabilizada.

\subsection{Selección de suelos}

En la totalidad de las normas se encuentran referencias a la selección de los suelos, aunque los planteamientos en cada uno de los documentos son diferentes e incluso algunas veces escasos $(26,30)$. Lo más usual son recomendaciones de resultados en base a determinados ensayos que en muchos de los casos son imprecisos, no cuantificables o basados en pruebas preliminares.

El grupo de propiedades más citadas son textura y plasticidad $(23,24,35,39,40,61)$ aunque también hay indicaciones sobre contenido orgánico y contenido de sales, sobre todo si ese suelo va a ser estabilizado. Los diagramas de plasticidad y textura son frecuentes en la norma francesa (24), colombiana (20) o española (23), mientras que en las normas brasileñas (7-19) nos recomiendan unos valores de límite líquido $(\leq 45 \%)$ y índice plástico $(\leq 18 \%)$ o los valores de las diferentes fracciones del suelo en otras normas como Perú (35) o Zimbabue (61). Otras propiedades relativas a la composición química del suelo o pH son inexistentes.
Otro enfoque es valorar el suelo conforme al producto de construcción que constituirá los muros (21,32-34). Esto se realiza porque no hay una relación directa entre el comportamiento del producto y las características del suelo utilizado. Se comprueba mediante los elementos construidos indicando pruebas a realizar y resultados requeridos según técnica utilizada (32-34) o comprobando los bloques directamente (21).

\subsection{Requisitos de los productos}

Trataremos a continuación el aspecto relativo a las propiedades exigibles para las piezas de adobe y de BTC y también para el caso de la tapia en el que el producto resultante de la construcción es el propio muro. Las especificaciones hacen referencia a la clasificación de productos, características dimensionales, geométricas, de aspecto, físico-químico o mecánicas-higrométricasfísicas mediante valores exigidos (36) o recomendados. Estas normas de producto que especifican los requisitos que debe satisfacer para establecer su aptitud al uso, son muy habituales en el marco normativo de tierra $(7,11,12,19,20,23,24,26,30,33$, $35,56,57,61)$.

En las normas los valores exigibles o recomendados se obtienen mediante ensayos, cuyo procedimiento se especifica en las mismas, convirtiéndose en normas de ensayo. Esto es así por no estar normalizadas previamente o suponer variaciones de las existentes. Son los casos NBR 8492 (8), NMAC 14.7.4 (21), XP P13-901 (24), IS 1725 (26), KS 02-1070 (30) NZS 4298 (33) o NTP 331.202 (37).

Las normas analizadas referentes a BTC se encuentran con una clasificación más amplia, mayores características geométricas o de aspecto que las referentes a piezas de adobe. Una clasificación muy usual para los tipos de BTC es según restricciones mecánicas (26), a partir de los valores de comprensión, caso de la norma colombiana (20), española (23) o en las normas ARSO (39-52). Suelen ser bloques sólidos pero se admiten hendiduras y perforaciones (7-19,39-52), existiendo limitaciones a esas perforaciones (33) o siendo condición necesaria (26).

La recepción de bloques comprimidos y adobe, incluyendo los requisitos exigibles, el muestreo a realizar y las condiciones de aceptabilidad se encuentran recogidos en NTP 331.203, (38), XP P13-901 (24), IS 1975 (26) y ARS 680 (39). También para el caso de la norma española (23) se exige garantizar todas las preinscripciones citadas, efectuando las verificaciones correspondientes según la norma. 


\subsection{Ensayos}

Gran parte de la información recogida en normas o reglamentos de edificación con tierra se refiere a procedimientos de ensayos. Hay publicaciones referidas exclusivamente a ensayos (8, 13,37,39-52); otras normas introducen los ensayos en anejos, como la IS 1725 (26), KS 02-1070 (30), NZS 4298 (33), SAZS 724 (61); mientras que otros documentos lo acompañan a normas de productos, caso de la norma peruana (35), francesa (24) y española (23).

La normativa de edificación con tierra es bastante autónoma y muchas veces se describen procedimientos de ensayos propios de técnicas constructivas con tierra, como los ensayos de erosión (33-61). También se encuentran muchas referencias a normas nacionales de otros campos del conocimiento, sobre todo en lo referente a preparación y clasificación de suelos. Entre estas referencias destacan la textura y plasticidad, como en las normas brasileñas (7-19), la SAZS 724 (61), NZS 4298 (33), XP P13-901 (24), y la norma española (23); determinación del contenido de materia orgánica $(23,30)$ o la determinación del contenido óptimo de humedad de un suelo. Para la absorción del agua en BTC se citan muchas normas nacionales referentes al ladrillo $(23,24,26)$.

Alguno de los ensayos más citados en las normativa de tierra cruda son de erosionabilidad, ciclos de humectación /secado y de resistencia a comprensión. Los ensayos de caída de agua o pulverizado a presión son bastantes homogéneos en cualquiera de las versiones realizadas $(23,26,33,61)$, mientras que los procedimientos de ensayos de ciclos de humectación y secado son muy dispares en lo referente a ciclos o tiempos de secado.

Los ensayos de resistencia a comprensión se consideran indicadores de la calidad de los elementos de tierra, pero se encuentran propuestas muy poco comparables. Si nos centramos en el ensayo a comprensión de las piezas de BTC nos encontramos con dos alternativas: ensayar el bloque partido y apilado, caso de normas brasileñas (7-19) y norma francesa (24); o ensayar la pieza com- pleta como en el caso de la norma española (23), ya que con la presencia del mortero se añade un nuevo parámetro además del propio de la pieza.

Existen también ensayos relacionados con el esfuerzo cortante mediante compresión diagonal (35) o ensayos de resistencia a tracción con módulo de rotura o a la tracción por flexión de elementos individuales (21, $30,32-35)$.

\section{CONCLUSIONES}

En la actualidad se evitan las normas con finalidades específicas: especificaciones, procesos de fabricación o métodos de ensayo, tendiéndose a una publicación conjunta de varias finalidades, tal y como se muestra en la norma española o francesa. La mayor parte de normas son normas de producto, ampliando su campo de aplicación a procedimientos de ensayos.

En la mayoría de los casos este tipo de documentos se refieren a una o dos técnicas. No se suelen utilizar normas y reglamentos que abarquen la construcción con tierra en su totalidad.

De las normas analizadas destacan las referidas a los bloques de tierra comprimida, para los que abunda la regulación, sobre todo en lo relativo a especificación del bloque, características geométricas, dimensionales, de aspecto, físico-químicas, etc.

El conjunto de normas de Nueva Zelanda presenta un modelo equiparable al enfoque actual que se da a otros materiales de construcción, caracterizado por la buena definición de las técnicas objeto, por el abundante desarrollo de todas las fases del proceso para las tres técnicas principales, y por la descripción minuciosa de los procedimientos de ensayo propuestos.

Es necesario homogeneizar los ensayos aplicados a las construcciones con tierra, tanto para piezas como para muros monolíticos, para poder realizar un adecuado análisis comparativo entre las diferentes técnicas de construcción existentes.

\section{BIBLIOGRAFÍA}

(1) Soronis, G.: "Standards for design life of buildings: utilization in the design process". Construction and Building Materials, Vol. 10 (1996) № I, pp. 487-490.

(2) Cañas Guerrero, I.; Jiménez Delgado, M.C.: "The selection of soils for unstabilizaed earth building: a normative review." Construction and building materials. Vol 21 (2007) pp. 237-251.

(3) Hooton, R.D.: "Bridging the gap between research and standards." Cement and Concrete Research, Vol 38 (2008), pp. 247-258. 
(4) Mahlia, T.M.I.; Saidur, R.: "A review on test procedure, energy efficiency standards and energy labels for room air conditioners and refrigerator-freezers." Renewable and Sustainable Energy Reviews, vol. 14 (2010) pp. 1888-1900.

(5) Martins Neves, C.; Villaça Coelho, A.C.: “Un paseo por las normas de construcción con tierra en los países iberoamericanos ". VIII ${ }^{\circ}$ Seminario Iberoamericano de construcción con tierra $I^{\circ}$ seminario argentino de arquitectura y construcción con tierra (2009).

(6) AENOR: Normalización y actividades relacionadas. Vocabulario general. UNE-EN 45020. (Guía ISO/IEC 2:2004) Madrid, 2007.

(7) ABNT: Tijolo maciço de solo-cimento. NBR 8491 EB1481. Associação Brasileira de Normas Técnicas, Rio de Janeiro, 1984.

(8) ABNT: Tijolo maciço de solo-cimento - Determinação da resistência à compressão e da absorção d’água. NBR 8492 MB1960. Associação Brasileira de Normas Técnicas, Río de Janeiro, 1984.

(9) ABNT: Fabricação de tijolo maciço de solo-cimento com a utilização de prensa manual. NBR10832 NB1221. Associação Brasileira de Normas Técnicas, Río de Janeiro, 1989.

(10) ABNT: Fabricação de tijolo maciço e bloco vazado de solo-cimento com utilização de prensa hidráulica. NBR 10833 NB1222. Associação Brasileira de Normas Técnicas, Río de Janeiro, 1989.

(11) ABNT: Bloco vazado de solo-cimento sem função strutural. NBR 10834 EB1969. Río de Janeiro: Associação Brasileira de Normas Técnicas. 1994.

(12) ABNT: Bloco vazado de solo-cimento sem função estrutural - Forma e dimensões. NBR 10835 PB1391. Associação Brasileira de Normas Técnicas, Río de Janeiro, 1994.

(13) ABNT: Bloco vazado de solo-cimento sem função estrutural - Determinação da resistência à compressão e da absorção de agua. NBR 10836 MB3072. Associação Brasileira de Normas Técnicas, Río de Janeiro, 1994.

(14) ABNT: Solo-cimento - Ensaio de compactação. NBR 12023 MB3359. Associação Brasileira de Normas Técnicas, Río de Janeiro, 1992.

(15) ABNT: Solo-cimento - Moldagem e cura de corpos-de-prova cilíndricos. NBR 12024 MB3360. Associação Brasileira de Normas Técnicas, Río de Janeiro, 1992.

(16) ABNT: Solo-cimento - Ensaio de compressão simples de corpos-de-prova cilíndricos. NBR 12025 MB3361. Associação Brasileira de Normas Técnicas, Río de Janeiro, 1990.

(17) ABNT: Solo-cimento - Ensaio de durabilidade por molhagem e secagem. NBR 13554. Associação Brasileira de Normas Técnicas, Río de Janeiro, 1996.

(18) ABNT: Solo-cimento - Determinação da absorção d'água. NBR 13555. Associação Brasileira de Normas Técnicas, Río de Janeiro, 1996.

(19) ABNT: Materiais para emprego em parede monolítica de solo-cimento sem função estrutural. NBR 13553. Associação Brasileira de Normas Técnicas, Río de Janeiro, 1996.

(20) ICONTEC: Bloques de suelo cemento para muros y divisiones. Definiciones. Especificaciones. Métodos de ensayo. Condiciones de entrega. NTC 5324. Instituto Colombiano de Normas Técnicas y Certificación, 2004.

(21) CID: New Mexico Earthen Buildings Materials Code. NMAC 14.7.4. 2003. Construction Industries Division CID of the regulation and Licensing Departament, Santa Fe, 2004.

(22) ASTM International: Standard Guide for Design of Earthen Wall Building Systems. E2392 M-10. Pennsylvania 19428-2959, United States, 2010.

(23) AENOR: Bloques de tierra comprimida para muros y tabiques. Definiciones, especificaciones y métodos de ensayo. UNE 41410, Madrid, 2008.

(24) AFNOR: Compressed earth blocks for walls and partitions: definitions - Specifications - Test methods - Delivery acceptance conditions. XP P13-901, Saint-Denis La Plaine Cedex, 2001.

(25) BIS: Code of practice for in-situ construction of walls, in building soil-cement. IS 2110 Bureau of Indian Standards, 1980.

(26) BIS: Specification for soil based blocks used in general building construction. IS 1725 Bureau of Indian Standards, 1982.

(27) BIS: Improving earthquake resistance of earthen buildings - Guidelines. IS 13827. Bureau of Indian Standards, 1993.

(28) Italia. Legge 24 Diciembre 2003, n. 378: “Disposizioni per la tutela e la valorizzazione dell'architettura rurale". Gazzetta Ufficiale, no 13 (2004).

(29) Italia. Regione Piemonte L.R. 2/06: "Norme per la valorizzazione delle costruzioni in terra cruda". B.U.R. Piemonte, $\mathrm{n}^{\mathrm{o}} 3$ (2006).

(30) KEBS: Specifications for stabilized soil blocks. KS02-1070:1993 (1999) Nairobi: Kenya Bureau of Standards, 1999.

(31) SON: Standard for stabilized earth bricks. NIS 369:1997. Lagos: Standards Organisation of Nigeria, 1997.

(32) SNZ: Engineering design of earth buildings. NZS 4297:1998. Wellington: Standards New Zealand, 1998.

(33) SNZ: Materials and workmanship for earth buildings. NZS 4298:1998. Wellington: Standards New Zealand, 1998. 
(34) SNZ: Earth buildings not requiring specific design. NZS 4299:1999. Wellington: Standards New Zealand, 1999.

(35) SENCICO: Adobe. NTE E 0.80. Reglamento Nacional de Construcciones, Lima, 2000.

(36) INDECOPI: Elementos de suelo sin cocer: adobe estabilizado con asfalto para muros: Requisitos. NTP 331.201. nstituto Nacional de Defensa de la Competencia y de la Protección de la Propiedad Intelectual, Lima, 1978.

(37) INDECOPI: Elementos de suelos sin cocer: adobe estabilizado con asfalto para muros: Métodos de ensayo. NTP 331.202. Instituto Nacional de Defensa de la Competencia y de la Protección de la Propiedad Intelectual, Lima, 1978.

(38) INDECOPI: Elementos de suelos sin cocer: adobe estabilizado con asfalto para muros: Muestra y recepción. NTP 331.203. Instituto Nacional de Defensa de la Competencia y de la Protección de la Propiedad Intelectual, Lima, 1978.

(39) ARSO: Compressed earth blocks, Standard for terminology. African Regional Standard 670: 1996 Nairobi, 1996.

(40) ARSO: Compressed Earth Blocks, Definition, classification and designation of compressed earth blocks. African Regional Standard 671: 1996 Nairobi, 1996.

(41) ARSO: Compressed Earth Blocks, Definition, classification and designation of earth mortars. African Regional Standard 672: 1996 Nairobi, 1996.

(42) ARSO: Compressed Earth Blocks. Definition, classification and designation of compressed earth blocks masonry. African Regional Standard 673:1996 Nairobi, 1996.

(43) ARSO: Compressed Earth Blocks. Technical specifications for ordinary compressed earth blocks. African Regional Standard 674: 1996Nairobi, 1996.

(44) ARSO: Compressed Earth Blocks-Technical specifications for facing compressed earth blocks. African Regional Standard 675: 1996 Nairobi, 1996.

(45) ARSO: Compressed Earth Blocks-Technical specifications for ordinary earth mortars.African Regional Standard 676: 1996 Nairobi, 1996.

(46) ARSO: Compressed Earth Blocks-Technical specifications for facing earth mortars. ARS 677: 1996 Nairobi, 1996.

(47) ARSO: Compressed Earth Blocks-Technical specifications for ordinary compressed earth block masonry. ARS 678: 1996 Compressed Earth Blocks-Technical specifications for ordinary compressed earth block masonry. Nairobi, 1996.

(48) ARSO: Compressed Earth Blocks-Technical specifications for facing compressed earth block masonry. ARS 679: 1996 Nairobi, 1996.

(49) ARSO: Compressed Earth Blocks. Code of practice for the production of compressed earth blocks. ARS 680: 1996 Nairobi, 1996.

(50) ARSO: Compressed Earth Blocks. Code of practice for the preparation of earth mortars. ARS 681: 1996 Nairobi, 1996.

(51) ARSO: Compressed Earth Blocks. Code of practice for the assembly of compressed earth block masonry. ARS 682: 1996 Nairobi, 1996.

(52) ARSO: Compressed Earth Blocks. Standard for classification of material identification tests and mechanical tests. ARS 683: 1996 Nairobi, 1996

(53) SLSI: Specification for compressed stabilized earth blocks. Part 1: Requirements SLS 1382-1. Sri Lanka Standards Institution, 2009.

(54) SLSI: Specification for compressed stabilized earth blocks. Part 2: Test Methods. SLS 1382-2. Sri Lanka Standards Institution, 2009.

(55) SLSI: Specification for compressed stabilized earth blocks. Part 3: Guidelines on production, design and construction. SLS 1382-3. Sri Lanka Standards Institution, 2009.

(56) INNORPI: Blocs de terre comprimée ordinaires - Spécifications techniques. NT 21.33:1996. Tunisian Standards, 1998.

(57) INNORPI: Blocs de terre comprimée - Définition, classification et désignation. NT 21.35:1996. Tunisian Standards, 1998.

(58) TSE: Cement Treated Adobe Bricks. TS 537. Turkish Standard Institution, Ankara, 1985.

(59) TSE: Adobe Blocks and Production Methods. TS 2514. Turkish Standard Institution, Ankara, 1997.

(60) TSE: Adobe Buildings and Construction Methods. TS 2515. Turkish Standard Institution, Ankara, 1985.

(61) SAZ: Standard Code of Practice for Rammed Earth Structures. SAZS 724:2001. Standards Association of Zimbawbe, Harare, 2001.

(62) Keable, J.: Rammed Earth Structures. A code of Practice. Intermediate Technology Publications, London, 1996. 